Journal of Computer Science 8 (9): 1547-1553, 2012

ISSN 1549-3636

(C) 2012 Science Publications

\title{
Fetal Electrocardiogram Extraction Using Let Techniques
}

\author{
${ }^{1}$ Hemajothi, S. and ${ }^{2}$ K. Helen Prabha \\ ${ }^{1}$ Department of ECE, St. Peter's University, Chennai, India \\ ${ }^{2}$ Department of ECE, RMD Engineering College, Chennai, India
}

\begin{abstract}
Fetal Electrocardiogram Extraction (FECG) identifies the congenital heart problems at the earlier stage. The major problem in the non invasive procedure is the extraction of FECG from Maternal ECG (MECG) and many interferences. The proposed methods (i) Combination of Adaptive Neuro Fuzzy Inference (ANFIS) and Fractional spline wavelet (ii) Combination of Fractional spline wavelet and ANFIS (iii) Combination of ANFIS and SURE-LET and (iv) Combination of SURE-LET and ANFIS remove the unwanted noises present in the FECG more effectively. This new approach extracts FECG by removing the noisy Abdominal ECG (AECG) and subsequently cancels the MECG. The pure thoracic ECG (TECG) or maternal ECG was used to remove noisy MECG present in the signal from abdomen signal and thereby the required noiseless FECG is extracted by means of the new approach. The excellence of the LET techniques are evaluated using Mean Square Error (MSE) and Peak Signal to Noise Ratio (PSNR). The result of combination of ANFIS and SURELET gives the best result and the closest match to the simulated FECG with high PSNR and low MSE among all the proposed methods.
\end{abstract}

Keywords: Abdominal Electrocardiogram (AECG), Adaptive Neuro-Fuzzy Inference System (ANFIS), Fetal electrocardiogram (FECG), Maternal Electro Cardiogram (MECG), Mean Square Error (MSE), Peak Signal to Noise Ratio (PSNR)

\section{INTRODUCTION}

The fetal Electrocardiogram (FECG) calculates fetal cardiac frequency and in the prediction of fetal acidosis. Medical treatment can be given to the mother at the earlier stages if any abnormalities are found and thereby congenital problems can be avoided. The ECG signals are taken by placing electrodes on the mother's thorax and abdomen. The ECG signals obtained from maternal abdomen are a combination of fetal ECG, maternal ECG, noises like electromyogram, power line interference, base line wander and other random noises. For the extraction of FECG, estimation from the abdominal signal and separation from the noises are essential.

Related works: Novel method for fetal ECG extraction from a combination of FECG, MECG and noises is proposed in (Khamene and Negahdaripour, 2000). The ECG taken from mother's thorax is original thoracic ECG or maternal ECG and the signal taken from abdomen area has mother's ECG and fetal ECG. The mother's ECG signal portion present in the abdominal signal is a nonlinear signal of mother's thoracic ECG. An efficient method of ANFIS and wavelet is proposed in (Swarnalatha and Prasad, 2010a; 2010b) to get the required fetal ECG from the signal in mother's abdomen. ANFIS technique for fetal ECG extraction is presented in (Assaleh, 2007). Hybrid technique of correlation and artificial neural network is presented in (Hasan et al., 2009). Adaptive neuro-fuzzy method (ANFIS) in (Vijila et al., 2006). FPGA implementation of Gamma filter for extracting FECG is presented in (HelenPrabha and Natarajan, 2007), FECG extraction using ANFIS with Gamma adaptations in (HelenPrabha and Valarmathy, 2007), the noises and the mother's ECG (MECG) present in the distorted form in the composite signal are removed by adaptive noise cancellation property of Gamma ANFIS (Vijila and Kanagasabapathy, 2005). Polynomial networks technique for the FECG extraction presented in (Assaleh and Al-Nashash, 2005). Hybridisation of Singular Value Decomposition (SVD) and ANFIS is discussed in (Al-Zaben and Al-Smadi, 2006).

\section{MATERIALS AND METHODS}

ANFIS: Adaptive neuro fuzzy inference system was discussed by Jang and Gulley (1993). Adaptive Noise Cancellation (ANC) using ANFIS technique is shown in Fig. 1. The unwanted noises can be removed by finding the non-linearity between the mother's ECG and the noises by ANC technique.

Here, $x(k)$ gives the fetal ECG signal obtained from the unwanted noisy signal. $\mathrm{n}(\mathrm{k})$ is the noise signal from mother's abdomen MECG. 


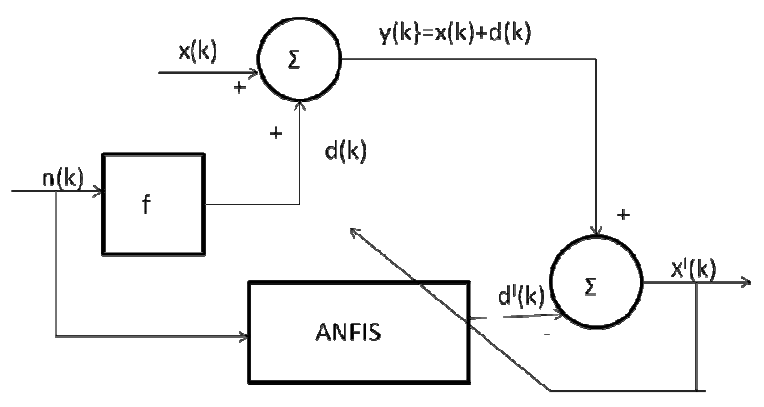

Fig. 1: Adaptive noise cancelling structure

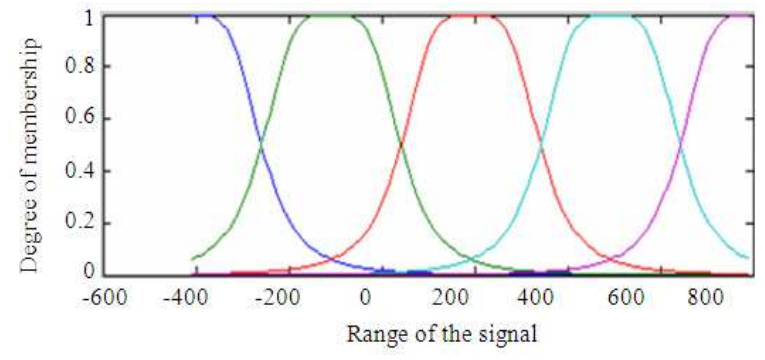

Fig. 2: Bell shape membership function

The noise signal goes through unknown nonlinear dynamics (f) and generates a distorted noise $\mathrm{d}(\mathrm{k})$, which is then added to $\mathrm{x}(\mathrm{k})$ to form the measurable output signal (abdominal) $y(k)$. The intention here is to differentiate $\mathrm{x}(\mathrm{k})$ from the abdominal signal $\mathrm{y}(\mathrm{k})$ which has the mixture of wanted signal $\mathrm{x}(\mathrm{k})$ and $\mathrm{d}(\mathrm{k})$ and the unwanted noisy signal.

ANFIS is a multilayer feed forward network. This architecture has five layers such as fuzzy layer, product layer, normalized layer, de-fuzzy layer and total output layer. The fixed nodes are represented by circle and the nodes represented by square are the parameters to be learnt. The system architecture of ANFIS is shown in Fig. 2. ANFIS gives the advantages of the mixture of neural network and fuzzy logic (Helenprabha and Natarajan, 2007). The aim of mixing fuzzy and neural networks is to design an architecture which uses a fuzzy logic to show knowledge in a fantastic manner, in addition to the learning nature of a neural network to maximize its parameters. The disadvantages of both the black box behavior of neural networks and the problems of getting appropriate membership values for fuzzy systems can be neglected.

Membership function in ANFIS: A Membership Function (MF) is a curve which explains how each and every point in the input space is mapped to a membership value (or degree of membership) between 0 and 1(Helenprabha and Natarajan, 2007). Here, generalized bell type MF is used for the FIS parameters tuning. This is shown in Fig. 2. It is specified by three parameters namely i a , i b and i c represents the width, centre and slope of the curve. The bell shaped functions change whenever there ia s change in the parameter value. Smoothness and concise notation is the major plus point of this technique.

FIS Structure and MF Parameter Adjustment: The very standard structure of a Fuzzy Inference System (FIS) maps input characteristics to input membership functions, input membership function to rules, rules to a set of output characteristics, output characteristics to output membership functions and the output membership function to a single-valued output or a decision associated with the output. In a conventional FIS, the number of rules are determined by an expert who is same as the target system. If there is no expert, emperical selection of membership function is given to each input. The FIS is applied to modeling systems whose rule structure is essentially predetermined by the user's interpretation of the characteristics of the variables in the model. Here, the shape of the membership functions depends on parameters and changing these parameters will change the shape of the MF. Instead of just looking at the data to choose the MF parameters, MF parameters can be chosen automatically using ANFIS. Hybrid learning algorithm is used in ANFIS to train the network parameters. It mixes the gradient method and the least square estimate to find the MF parameters.

Gradient method is normally slow and become strucked in local minima. So, hybrid rule is used to decrease the area of the search space in the gradient method and also reduces the convergence time. As a result of multilayer ANFIS architecture, the parameters in the hidden layer is tuned by the gradient method learning rule and the least squares method is used to find the parameters in the output layer.

ANFIS architecture: For simplicity, assume that the fuzzy inference system has two inputs $x$ and $y$ and one output z. A first-order (Takagi and Sugeno, 1985) fuzzy model has the following rules:

- Rule 1: If $\mathrm{x}$ is $\mathrm{A} 1$ and $\mathrm{y}$ is $\mathrm{B} 1$, then $\mathrm{f} 1=\mathrm{p} 1 \mathrm{x}+\mathrm{q} 1 \mathrm{y}+\mathrm{r} 1$

- Rule 2: If $x$ is A2 and $y$ is B2, then $f 2=p 2 x+q 2 y+r 2$

Here, $\mathrm{x}$ is $\mathrm{A} 1$ and $\mathrm{y}$ is $\mathrm{B} 1$ and $\mathrm{x}$ is $\mathrm{A} 2$ and $\mathrm{y}$ is $\mathrm{B} 2$ are called as the premise section (non linear section), while $\mathrm{f1}$ $=\mathrm{p} 1 \mathrm{x}+\mathrm{q} 1 \mathrm{y}+\mathrm{r} 1$ and $\mathrm{f} 2=\mathrm{p} 2 \mathrm{x}+\mathrm{q} 2 \mathrm{y}+\mathrm{r} 2$ are called as the consequent section (linear section). i.e., p1, p2, q1, q2, r1, r2 are linear parameters and A1, A2, B1, B2 are non linear parameter. The corresponding equivalent ANFIS architecture is shown in Fig. 3 (Jang and Gulley, 1993). 


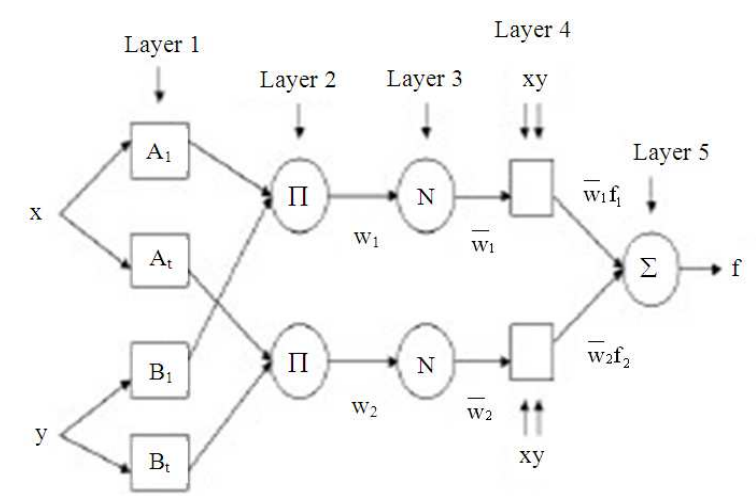

Fig. 3: ANFIS architecture

ANFIS is a multilayer feed forward network. The system architecture consists of five layers namely, fuzzy layer, product layer, normalized layer, de-fuzzy layer and total output layer. The fixed nodes are represented by circle and the nodes represented by square are the parameters to be learnt. The forthcoming section gives a clear detail about the link between the input and output of each layer in ANFIS.

Layer 1: It is the fuzzy layer. Every node $i$ in this layer is an adaptive node with a node function. $\mathrm{O}_{\mathrm{l}, \mathrm{i}}$ is the output of the ith node of the layer 1 which is shown in Eq. 1:

$$
\begin{aligned}
& \mathrm{O}_{1, \mathrm{i}}=\mu_{\mathrm{Ai}}(\mathrm{x}) \text { for } \mathrm{i}=1,2, \text { or } \\
& \mathrm{O}_{1, \mathrm{i}}=\mu_{\mathrm{Bi}-2}(\mathrm{y}) \text { for } \mathrm{i}=3,4
\end{aligned}
$$

$\mathrm{x}$ (or $\mathrm{y}$ ) is the input node $\mathrm{i}$ and $\mathrm{Ai}$ (or $\mathrm{Bi}-2$ ) is a linguistic label associated with this node. Therefore $\mathrm{O}_{1, \mathrm{i}}$ is the membership grade of a fuzzy set (A1, A2, B1, B2). In this work, bell shaped Membership Functions (MF) are chosen and it is shown in Eq. 2:

$$
\mu_{\mathrm{A}}(\mathrm{x})=\frac{1}{1+\left|\frac{\mathrm{x}-\mathrm{c}_{\mathrm{i}}}{\mathrm{a}_{\mathrm{i}}}\right|^{2 \mathrm{~b}}}
$$

where, ai, bi and ci is are the premise parameter set to be learnt.

Layer 2: It is the product layer that consists of two nodes labeled as $\Pi$. The output is the product $(\mathrm{X})$ of all the incoming signals which is shown in Eq. 3:

$\mathrm{O}_{2, \mathrm{i}}=\mathrm{w}_{\mathrm{i}}=\left(\mu_{\mathrm{Ai}}(\mathrm{x})\right) \mathrm{X}\left(\mu_{\mathrm{Bi}}(\mathrm{y})\right) \mathrm{i}=1,2$
Each node represents the firing strength of the rule. $\mathrm{w}_{1}, \mathrm{w}_{2}$ are the weight functions of the next layer.

Layer 3: It is the normalized layer. Every node in this layer is a fixed node labeled Norm. Its function is to normalize the weight function with the following condition, where $\mathrm{O}_{3, \mathrm{i}}$ denotes the Layer 3 output as shown in Eq. 4:

$$
\mathrm{O}_{3, \mathrm{i}}=\overline{\mathrm{w}}_{\mathrm{i}}=\frac{\mathrm{w}_{\mathrm{i}}}{\mathrm{w}_{1}+\mathrm{w}_{2}}
$$

Outputs are called normalized firing strengths.

Layer 4: It is the defuzzy layer. Every node $\mathrm{i}$ in this layer is an adaptive node. The defuzzy relationship between the input and output of this layer can be defined below, where $04, \mathrm{i}$ denotes the layer 4 output as shown in Eq. 5:

$\mathrm{O}_{4, \mathrm{i}}=\overline{\mathrm{w}}_{\mathrm{i}} \mathrm{f}_{\mathrm{i}}=\overline{\mathrm{w}}_{\mathrm{i}}\left(\mathrm{p}_{\mathrm{i}} \mathrm{x}+\mathrm{q}_{\mathrm{i}} \mathrm{y}+\mathrm{r}_{\mathrm{i}}\right)$

where, $\overline{\mathrm{w}}_{\mathrm{i}}$ is the normalized firing strength from layer 3. $\mathrm{p}_{\mathrm{i}}, \mathrm{q}_{\mathrm{i}}$, ri denote the linear parameters which are also called consequent parameters of the node.

Layer 5: It is the total output layer, whose node is labeled as sum, which computes the overall output as the summation of all incoming signals. The result can be written as follows where as $\mathrm{O}_{5, \mathrm{i}}$ denotes the layer 5 output as shown in Eq. 6:

Overalloutput $=\mathrm{O}_{5,1}=\sum_{\mathrm{i}} \overline{\mathrm{w}}_{\mathrm{i}} \mathrm{f}_{\mathrm{i}}=\frac{\sum_{\mathrm{i}} \mathrm{w}_{\mathrm{i}} \mathrm{f}_{\mathrm{i}}}{\sum_{\mathrm{i}} \mathrm{w}_{\mathrm{i}}} \mathrm{i}=1,2$

The mixture of gradient descent method and least square method trained the ANFIS. Each epoch of hybrid learning consists of forward pass and backward pass. Least-square method is used to find the consequent parameters on the layer 4 during forward pass. Gradient descent method upgrades the premise parameters and propagates the error backward during the reverse pass (Jang and Gulley, 1993). The evalfis command determines the output of the FIS system for given input. Here, the reference signal MECG and the desired abdominal signals are given as a training pair for ANFIS.

Wavelet transform: The wavelet transform is a timescale representation technique, which explains a signal by means of correlation with translation and dilation of a function called as mother wavelet. The Discrete 
Wavelet Transform (DWT) is defined by splitting $\mathrm{f}(\mathrm{t})$ in to smaller non overlapping parts $\mathrm{f}_{\mathrm{i}}(\mathrm{t})$, taking a finite number of scales $\mathrm{N}$ and down sampling the discrete wavelet coefficients samples to $M$, the number of samples of $\mathrm{f}_{\mathrm{i}}(\mathrm{t})$ :

$$
\operatorname{DWT}_{i}(s, t, N)=\left\langle\sum_{j=1}^{N} \sum_{k-1}^{N} \operatorname{CWT}_{i}\left(s_{j}, \tau_{k}\right)\right\rangle \Downarrow N
$$

The wavelet denoising method consists of applying DWT to the original signal, thresholding the detail and approximation coefficients and inversing the threshold coefficients to obtain the time domain denoised data (Paraschiv-Ionescu et al., 2002). The performance of the wavelet denoising depends upon the type of wavelet transform, type of the wavelet, thresholding rule and the number of decomposition levels.

Surelet Denoising: The proposed new methodology to signal denoising, relies on the image-domain minimization of an estimate of the mean squared errorStein's Unbiased Risk Estimate (SURE). The denoising methods are explained as a linear combination of denoising processes and Linear Expansion of Thresholds (LET). According to the SURE and LET principles, denoising algorithm is particularly meant for solving a linear system of equations which is efficient and fast. By taking threshold over the undecimated Haar wavelet coefficients, good results are obtained which clearly explains a SURELET algorithm is more efficient.

SURE is nothing but an statistical estimate of the Mean Squared Error (MSE) between an original unknown signal and a noisy signal. This method relies upon the served data and never expects previous assumption on the noise-free signal.

The only statistical assumption is done on the noise.

Denoting by $\hat{v}$, an estimate of the noise-free video $\mathrm{v}$, the global MSE is expressed as:

$$
\begin{aligned}
& \text { MSE }=\frac{1}{N T} \underbrace{\sum_{t=1}^{T} \sum_{n=1}^{N} e_{j}^{T}(\hat{v} n-v n)(\hat{v} n-v n)^{T} e_{t}}_{\text {N } \times M S E_{t}} \\
& =\frac{1}{N T} \underbrace{\sum_{j=1}^{T} \sum_{t}^{j} \sum_{n=1}^{N^{j}} e_{t}^{T}\left(\hat{x}_{n}^{j}-x_{n}^{j}\right)\left(\hat{x}_{n}^{j}-x_{n}^{j}\right)^{T} e_{t}}_{N^{j} \times M_{t=1}}
\end{aligned}
$$

where, $e^{T} \hat{t} x^{j} n=\theta^{j} t\left(y^{j} n, p^{j} n\right)$ is the nth pixel of the $j$ th wavelet subband of the denoised frame t. It is obtained by thresholding the nth pixel of the jth wavelet subband of the noisy frame $t$, taking into account (some of) its neighboring frames. The subband superscript " $\mathrm{j}$ " and the time frame indication " $\mathrm{t}$ " can be removed for getting the clear idea. While taking the multiframe processing $\theta$ into our account: $\mathrm{R}^{\mathrm{T}} \times \mathrm{R}^{\mathrm{T}} \rightarrow \mathrm{R}$, the mean square error of wavelet sub-band $\mathrm{j}$ of frame $\mathrm{t}$ can be expressed without bias by:

$$
\in=\frac{1}{N} \sum_{n=1}^{N}\left[\left(\theta\left(y_{n}, p_{n}\right)-e_{t}^{T} y_{n}\right)^{2}+2 e_{t}^{T} R \Delta_{1} \theta\left(y_{n}, p_{n}\right)-N e_{t}^{T} R_{t}\right]
$$

Here, $\mathrm{p}^{\mathrm{n}}$ denotes any random variables statistically independent of $\mathrm{y}_{\mathrm{n}}$ and 1 corresponds to the gradient operator with respect to the first variable of the function, $\mathrm{y}_{\mathrm{n}}$.

Linear Expansion of Thresholds (LET): The thresholding function is expressed by a linear combination of thresholding functions, which was coined LET, that is:

$$
\theta\left(y_{n}, p_{n}\right)=\underbrace{\left[a_{1}^{T} a_{2}^{T} \ldots a_{k}^{T}\right]}_{a^{T}} \underbrace{\left[\begin{array}{l}
\theta_{1}\left(y_{n}, p_{n}\right) \\
\theta_{2}\left(y_{n}, p_{n}\right) \\
\theta_{n} \\
\theta_{K}\left(y_{n}, p_{n}\right)
\end{array}\right]}_{\theta\left(y_{n}, p_{n}\right)}
$$

where, a and $\theta$ are both $K T \times 1$ vectors. Each $\theta_{k}: R^{T} \times R^{T}-$ $\rightarrow \mathrm{R}^{\mathrm{T}}$ is an arbitrary vector-valued thresholding. The optimal-in the minimum-sense-parameters of (5) are the solution of a linear system of equations:

$\mathrm{a}_{\mathrm{opt}}=\mathrm{M}^{-1} \mathrm{C}$

Where:

$$
\left\{\begin{array}{l}
M=\sum_{n=1}^{N} \theta\left(y_{n}, p_{n}\right) \theta\left(y_{n}, p_{n}\right)^{T} \\
C=\sum_{n=1}^{N}\left(\theta\left(y_{n}, p_{n}\right)_{n}^{T}-\left(\Delta_{1} \theta\left(y_{n}, p_{n}\right)^{T} R\right) e_{t}\right)
\end{array}\right.
$$

The steps for the SURELET denoising are as follows:

Step 1: Obtain the noisy abdominal signal

Step 2: Set the number of iterations.

Step 3: Compute the standard deviation of the noise.

Step 4: Compute the Surelet transform of the signal.

Step 5: Denoise the signal using the calculated Parameters.

Step 6: Reconstruct to obtain the denoised signal. 
FECG extraction using SURELET and ANFIS: In this process, the ECG taken from mother's abdomen is pre-processed at first by surelet transform and the output is passed through ANFIS. The other input to ANFIS is the signal from the thorax area. The required FECG is the result from the ANFIS.

FECG Extraction Using ANFIS and SURELET: In this process, the signal mother's abdomen and from the thorax area is first passed through ANFIS to get the estimated TECG signal and the output is processed by SURELET transform to get the extracted FECG.

Fractional Spline Wavelets: Fractional spline wavelets are also called as splinelet. The Schoenberg's family of polynomial splines with uniform knots are extended to all fractional degrees. These splines, which involve linear combinations of the one sided power functions $\mathrm{x}+\mathrm{x} \alpha=\{\} \alpha \max 0$, are $\alpha$-Holder continuous for $\alpha \geq 0$. We construct the corresponding B-splines by taking fractional finite differences and provide an explicit characterization in both time and frequency domains.

The name Fractional splines indicates that it should have an approximation in fractional order, a property which have not been encountered before approximation theory. Absolutely, the error of approximation decays like $\mathrm{f}$ Pf $\mathrm{Oa} \mathrm{a}-=(\alpha+1)$ as $\mathrm{a} \rightarrow 0$. The asymptotic development of the L2-error is provided and quantitative error bounds are given to substantiate this issue.

The Strang-Fix theory is not satisfied by the fractional splines for non-integer $\alpha$ which gives the equivalence between the reproduction of polynomials of degree $\mathrm{n}$ and the order of approximation which is given by one degree more $(L=n+1)$. Particularly, the fractional splines will reproduce polynomials of degree $n$ with $n$ $1<\alpha \leq n$ while the order of approximation is $\alpha+1$.

The valid multiresolution analyses of L2 for $\alpha>-12$ is generated by fractional B-splines. But, for $\alpha<<-12$, the factor $(1+z)$ is not present in the refinement filters $\mathrm{H}(\mathrm{Z})$ which is essential for the building up of real wavelet bases. But, the filters have the correct fading property: $\mathrm{H}(\mathrm{e} \mathrm{j} \pi)=0$, which assures the segregation of unity condition (everywhere, excluding the knots).

The multiresolution analysis of L2 is satisfied by the above mentioned functions and so it can be used to construct new wavelet bases families with a parameter in a continuously-varying mode.

The steps for the Fractional spline wavelet denoising are as follows:

Step 1: Obtain the noisy abdominal signal.

Step 2: Decompose the signal into low frequency and high Frequency bands using splinelet transform.
Step 3: Analyze the bands to identify the high frequency noise.

Step 4: Remove the noise by replacing those bands with null bands.

Step 5: Reconstruct to get the noiseless signal.

FECG Extraction Using Splinelet and ANFIS: In this method, the abdominal ECG is first pre-processed by Fractional spline wavelet transform and the output is passed through ANFIS. The other input to ANFIS is the thoracic signal. The output of ANFIS is the extracted fetal ECG.

FECG Extraction Using ANFIS and Splinelet: In this method, the abdominal ECG and thoracic ECG is first passed through ANFIS to get the estimated thoracic signal and the output is processed by Fractional spline wavelet transform to get the extracted FECG.

\section{RESULTS}

The required FECG signal was obtained by the methods given below:

- ANFIS

- $\quad$ SURELET and ANFIS

- ANFIS and SURELET

- Fractional spline wavelet and ANFIS

- ANFIS and Fractional spline wavelet

Analysis of Method I: Simulated maternal ECG, Fetal ECG, Abdominal signal is used to extract original FECG. Figure 4 shows the signal from mother's abdomen, estimated signal from thorax area and the wanted FECG by ANFIS method. In this process, there is an oscillatory phenomena can be seen in the position of maternal ECG in the acquired signal.

The calculated MSE and PSNR values are as below.

MSE $=7.6518 \mathrm{e}-04$

$\mathrm{PSNR}=79.2932$

Analysis of Method II: The simulated abdominal signal is denoised using SURELET transform technique and the denoised signal is fed into ANFIS for FECG extraction. The output are depicted in Fig. 5.

The calculated MSE and PSNR values are as below.

$\mathrm{MSE}=84.5846$

$\mathrm{PSNR}=2.2627 \mathrm{e}-04$ 

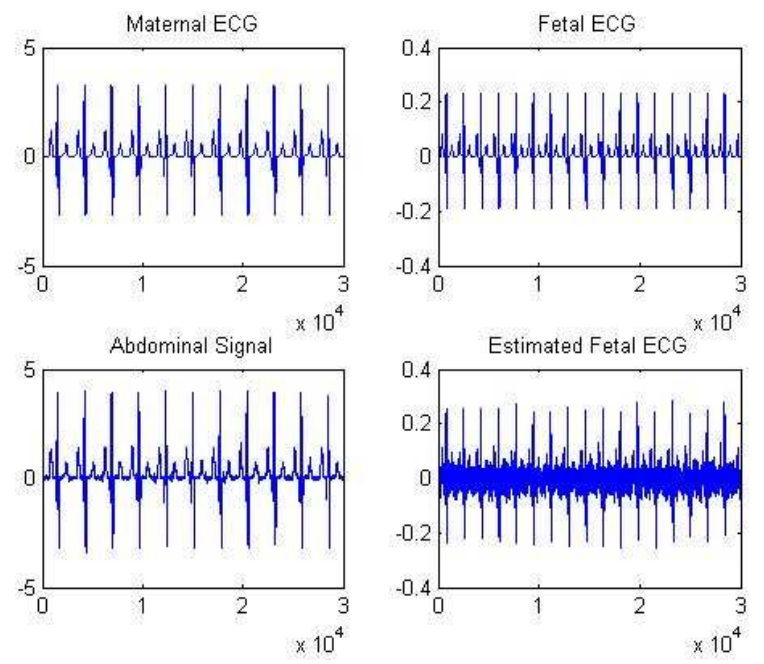

Fig. 4: FECG extraction using ANFIS
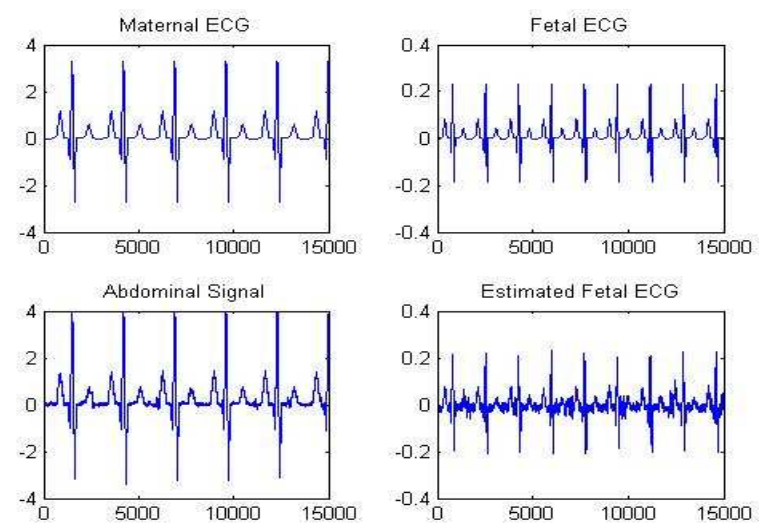

Fig. 5: FECG extraction using SURELET and ANFIS
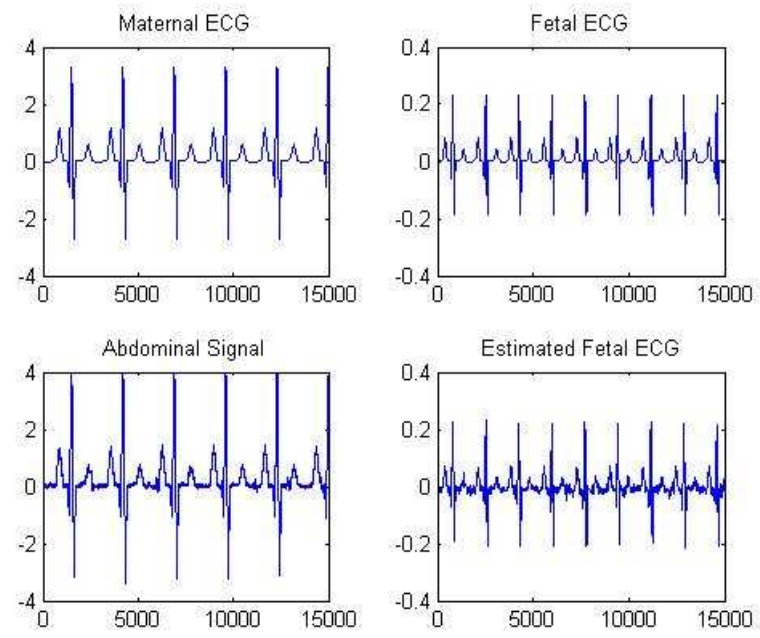

Fig. 6: FECG extraction using ANFIS and SURELET
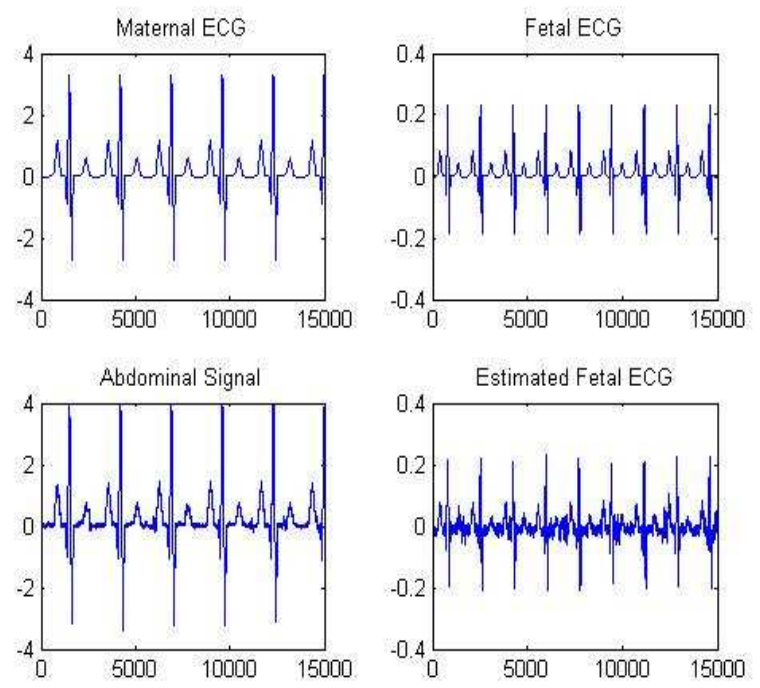

Fig. 7: FECG extraction using Splinelet and ANFIS
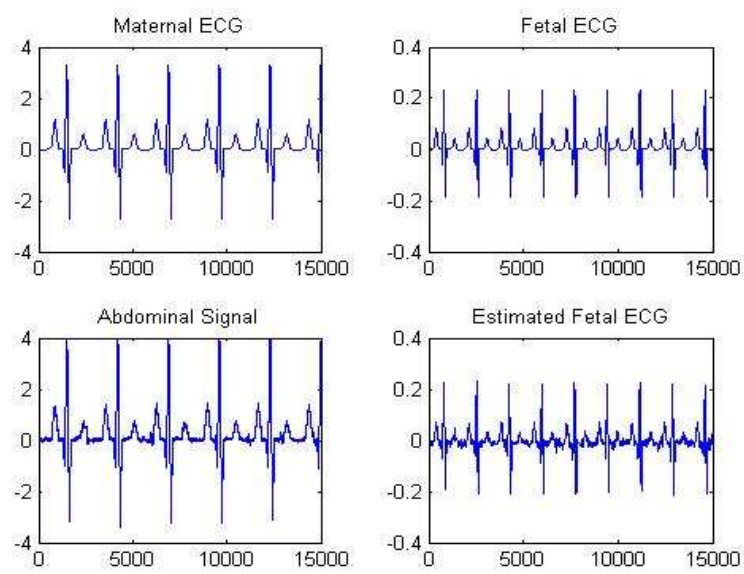

Fig. 8. FECG extraction using ANFIS and Splinelet

Analysis of Method III: SURELET post-processing is done by denoising the extracted FECG using the ANFIS. The results are shown in Fig. 6. Upon comparison, the SURELET post-processing provides more accurate fetal ECG extraction.

The calculated MSE and PSNR values are as below.

MSE $=2.1542 \mathrm{e}-04$

$\mathrm{PSNR}=84.7979$

Analysis of Method IV: In this process, the fractional spline transform is used to remove the random noise in the signal taken from mother's abdomen and the required fetal ECG is obtained by ANFIS as shown in Fig. 7. 
Table 1: Performance evaluation results

\begin{tabular}{lcl}
\hline Method & MSE & PSNR \\
\hline Surelet pre-processing & 84.5846 & $2.26 \mathrm{E}-04$ \\
Surelet post-processing & 84.7979 & $2.15 \mathrm{E}-04$ \\
Splinelet pre-processing & 83.1252 & $3.17 \mathrm{E}-04$ \\
Splinelet post-processing & 84.7703 & $2.17 \mathrm{E}-04$ \\
\hline
\end{tabular}
below.

The calculated MSE and PSNR values are as

MSE $=3.1663 \mathrm{e}-004$

$\mathrm{PSNR}=83.1252$

Analysis of method V: In this method, the signal from mother's abdomen is is estimated by ANFIS and fractional spline wavelet transform is used to extract FECG as shown in Fig. 8.

The calculated MSE and PSNR values are as below.

MSE $=2.1679 \mathrm{e}-004$

$\mathrm{PSNR}=84.7703$

\section{DISCUSSION}

The analysis of new mehodologies are particularly towards the efficiency of the required fetal ECG. The important parameters used for maintaining the efficiency of the fetal ECG are Peak Signal to Noise Ratio (PSNR) and mean square error (MSE). The results of performance evaluation for all the above mentioned methods are shown in the Table 1. Upon comparison, Post-processing using SURELET achieved the best results and can be concluded as the better of the five methods.

\section{CONCLUSION}

In this study, we have projected a new approach of hybrid algorithm of ANFIS and LET to obtain fetal ECG from mother's abdomen with high PSNR. ECG from the mother's abdominal area and from the thoracic area is used for obtaining the wanted fetal ECG signal. The proposed methods are evaluated using MSE and PSNR. While comparing the results, ANFIS followed by SURELET post-processing produces high PSNR which shows that this method produces good quality FECG signal. Further optimization can be performed for the better estimation of the wanted fetal ECG. The results will be used for hardware implementations.

\section{REFERENCES}

Al-Zaben, A. and A. Al-Smadi, 2006. Extraction of foetal ECG by combination of singular value decomposition and neuro-fuzzy inference system. Phys. Med. Biol., 51: 137-143. PMID: 16357436
Assaleh, K. and H. Al-Nashash, 2005. A novel technique for the extraction of fetal ECG using polynomial networks. IEEE Trans. Biomed. Eng., 52: 1148-1152. DOI: 10.1109/TBME.2005.844046

Assaleh, K., 2007. Extraction of fetal electrocardiogram using adaptive neuro-fuzzy inference systems. IEEE Trans. Biomed. Eng., 54: 59-68. PMID: 17260856

Hasan, M.A., M.I. Ibrahimy and M.B.I. Reaz, 2009. An efficient method for fetal electrocardiogram extraction from the abdominal electrocardiogram signal. J. Applied Sci., 5: 619-623.

Helenprabha, K. and A.M. Natarajan, 2007. FPGA implementation of gamma filter for extracting fetal electrocardiogram. IETE J. Res., 53: 433-440.

HelenPrabha, K. and S. Valarmathy, 2007. Fetal electrocardiogram using anfis with gamma adaptations. Int. J. HIT Tran. ECCN, 2: 427-434.

Jang, J.S.R., 1993. ANFIS: adaptive-network-based fuzzy inference system. IEEE Trans. Syst. Man Cybern, 23: 665-685. DOI: 10.1109/21.256541

Khamene, A. and S. Negahdaripour, 2000. A new method for the extraction of fetal ECG from the composite abdominal signal. IEEE Trans. Biomedical Eng., 47: 507-516. DOI: $10.1109 / 10.828150$

Paraschiv-Ionescu, A., C. Jutten, K. Aminian, B. Najafi and P. Robert, 2002. Source separation in strong noisy mixtures: A study of wavelet denoising preprocessing. IEEE Int. Conf. Acoustics Speech Signal Process., 2: 1681-1684.

Swarnalatha, R. and D.V. Prasad, 2010a. A novel technique for extraction of FECG using multi stage adaptive filtering. J. Applied Sci., 10: 319-324.

Swarnalatha, R. and D.V. Prasad, 2010b. Maternal ECG cancellation in abdominal signal using ANFIS and wavelets. J. Applied Sci., 10: 868-877. DOI: $10.3923 /$ jas.2010.868.877

Takagi, T. and M. Sugeno, 1985. Fuzzy identification of systems and its applications to modeling and control. IEEE Trans. Syst. Man Cybermetics, 15: 116-132.

Vijila, C.K.S. and P. Kanagasabapathy, 2005. Adaptive neuro fuzzy Inference System for extraction of fECG. IEEE INDICON. DOI: 10.1109/INDCON.2005.1590160

Vijila, S.C.K., M.E.P. Kanagasabapathy and S. Johnson, 2006. Fetal ECG extraction using softcomputing technique. J. Applied Sci., 6: 251-256. 